\title{
Simulation Experiment on Acoustic Emission of Pipeline Leakage
}

\author{
Wang Xin-hua ${ }^{1,2}$, Yang Jie ${ }^{1}$, Jiao Yu-lin ${ }^{2}$ and Niu Yong-chao ${ }^{3}$ \\ ${ }^{1}$ Wuhan University of Technology, Wuhan 430063, China; \\ ${ }^{2}$ Shangqiu Vocational and Technical College, Shangqiu 476000, China; \\ ${ }^{3}$ Shangqiu Jinpeng Industrial Co., Ltd., Shangqiu 476000, China \\ gyxxwxh@163.com
}

\begin{abstract}
With the heater pipeline leakage experiment conducted on the experiment table, inner pipeline leakage of heater was studied through the leakage failure detection system. The frequency distribution and the amplitude of acoustic emission signals of leakage versus internal pressure were analyzed. Variations of signals versus leakage aperture and dissemination distance were summarized. According to the mapping relationship between the leaking spot and acoustic emission signals, and between the leakage flow and acoustic emission signals, reasons of leakage failure were concluded. The results would be applied to heater leakage failure detection. And an on-the-spot detection in the power plant was conducted to test the heater under pressure in order to verify the results.
\end{abstract}

Keywords: acoustic emission; pipeline; leakage; detection; acoustic emission signals

\section{Introduction}

Heater is one of the jacked lines, usually applied to heat exchanger such as highlow heat exchanger in the power plant. It can also serve for chemical production, such as high-low reactor. A heater consists of inner pipeline and outer pipeline. The transmission media in the inner pipeline of the heater is water or raw materials while that in the outer pipeline, vapor, hot water, refrigerant or PCB carrier. Due to aging, crack, media erosion, environment erosion, wear and flaws, the transmission media in the inner pipeline of the heater are likely to leak. Acoustic emission of pipeline leakage is influenced by outer pipeline, substance between the inner and the outer pipeline, fluid state and interaction of fluids, the mechanism of which is very complex [1].

The sound wave emitted due to inner pipeline leakage is not a special phenomenon, because the pipe wall only acts as guided wave and doesn't release energy. But the gas leak at the leaking spot would excite the stress wave that could describe the structure of the material. So, it is a generalized acoustic emission phenomenon [2-6].

Waveform and frequency spectrum of acoustic emission signals produced from inner pipeline leakage versus pressure, leakage aperture and transmission distance were studied with the removal of the shell through experiment.

\section{The Test System}

Online leakage failure detection system and device of acoustic emission consist of two parts 


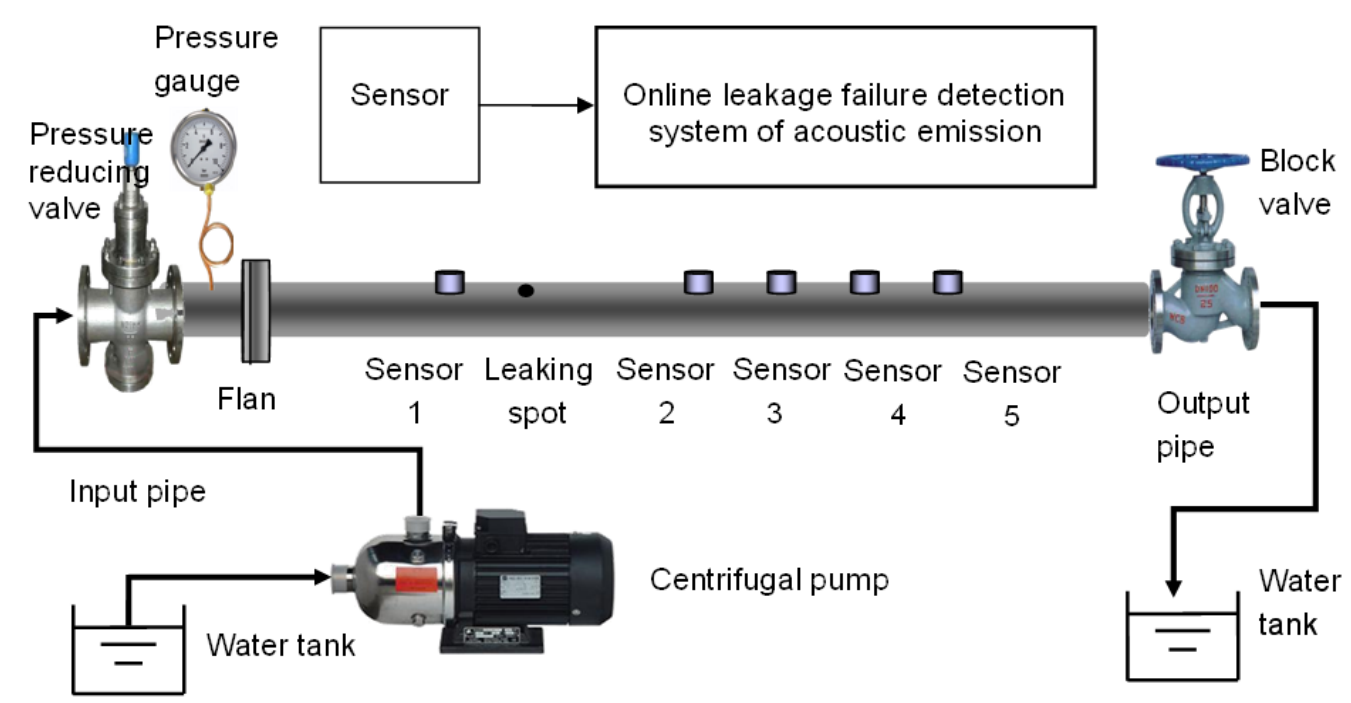

Figure1. Simulation system of pipeline leakage

\section{(1) Online Leakage Failure Detection System of Acoustic Emission}

As was introduced in Chapter 4, hardware included sensor, pre-amplifier, signal acquisition card, device and data processing and analysis system. The signal acquisition card was the AEDSP2 $32 / 16$ made in U.S. The sensor was the singleended broadband $\mathrm{R} 15$, produced by $\mathrm{PAC}$, with the band ranging from $50 \mathrm{kHz} \sim 1 \mathrm{MH}$. The pass-band fluctuation was smaller than $30 \mathrm{~d}$ and its sensitivity was $120 \mathrm{~dB}$. To make the sensor the pipeline fit, coupling agent was smeared on the surface of the sensor and a magnetic installation seat was used to ensure the tightness.

\section{(2) Simulation system of pipeline leakage}

The seamless pipeline was $12 \mathrm{~m}$ in length, $5 \mathrm{~mm}$ in wall thickness and of ${ }^{\phi} 90 \mathrm{~mm}$ outside diameter. Pressure reducing valve and pressure gauge were installed at the input pipe. Block valve was installed at the output pipe. Centrifugal pump was responsible for supplying water and producing pressure. Details are shown in Table 1.

\section{Experiment}

(1) Noise signals during normal operation of the pipeline and acoustic emission signals under leakage were collected. Waveform and frequency spectrum of noise signals and acoustic emission signals were carefully studied to determine whether the leakage occurred [7-10].

(2) At the same leaking spot, leakage signals were collected under different internal pressures. Waveform and frequency spectrum versus pressure were studied. At $1 \mathrm{~mm}$, the inner pressure was increased from $0.2 \mathrm{MPa}$ to $0.5 \mathrm{MPa}$. Acoustic emission signals of leakage were collected for adding every $0.05 \mathrm{MPa}$.

(3) Under the same pressure, the leakage aperture was changed. The leaking spot was increased from $1 \mathrm{~mm}$ to $5 \mathrm{~mm}$. Acoustic emission signals of leakage were collected for adding every $1 \mathrm{~mm}$.

(4) Under the same pressure and the leakage aperture, the distance between the leaking spot and the sensor was changed. Leakage signals were collected to study the variation of signals versus transmission distance. The distance was increased 
from $1 \mathrm{~m}$ to $5 \mathrm{~m}$ and each time $1 \mathrm{~m}$ was added. The internal pressure was adjusted at a proper time. Dispersion of acoustic emission signals of leakage was studied on the basis of data collected

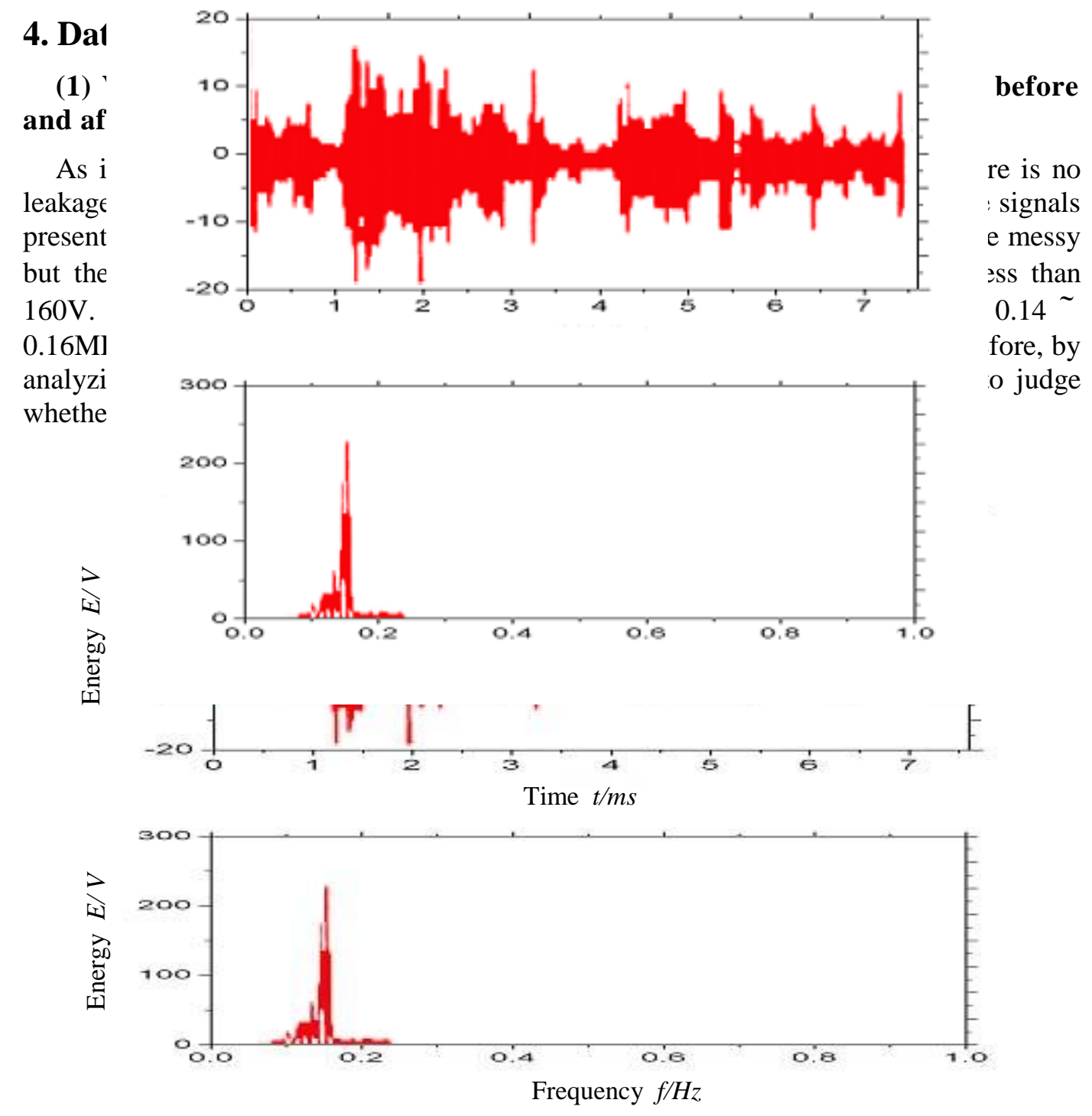

Figure 2.

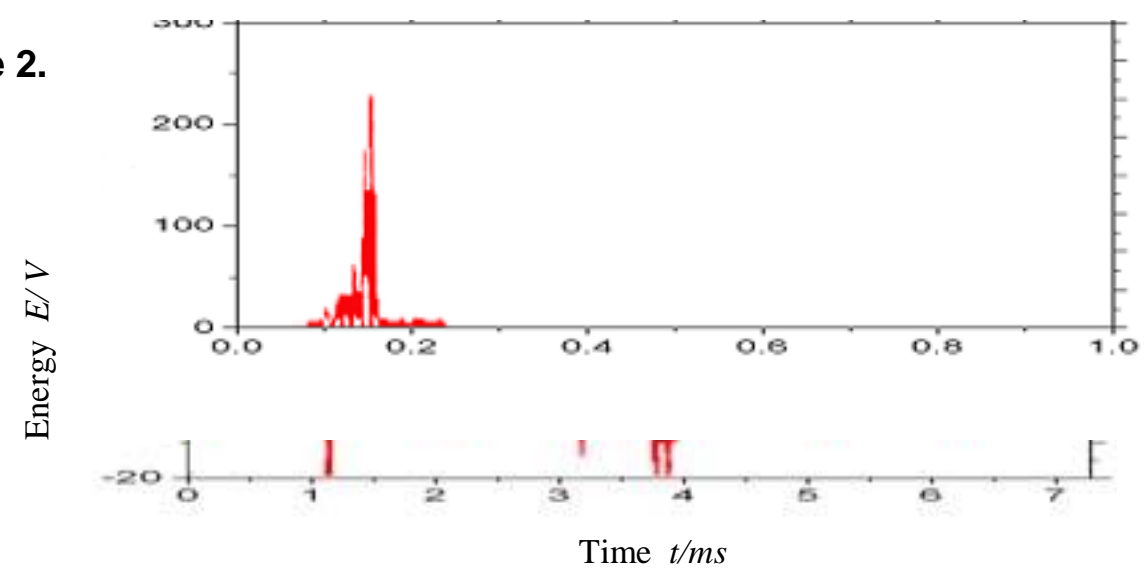




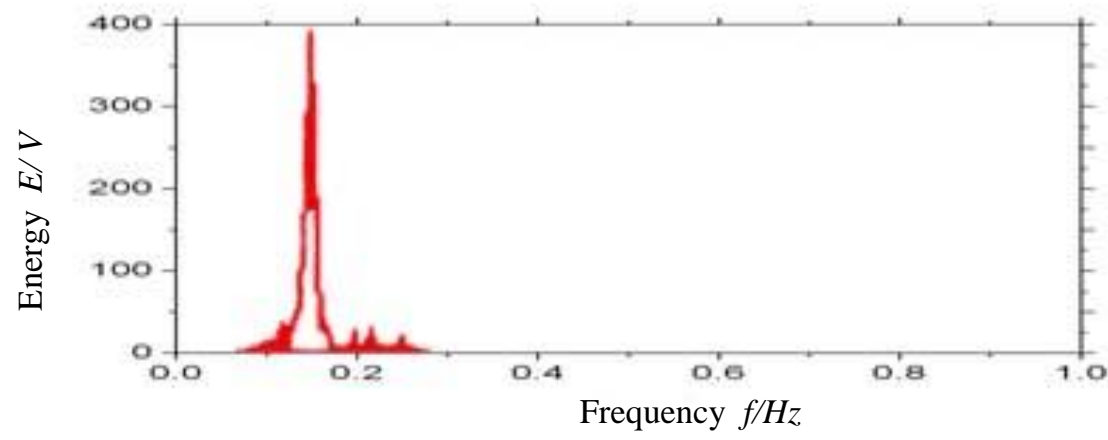

Figure 3. Acoustic Emission Signals and the Spectrum Under Leakage

(2) Variation of Waveform and Frequency Spectrum of Leakage Signals versus Internal Pressure at the Same Leaking Spot

Figure 4 shows waveform and frequency spectrum of leakage signals at $1 \mathrm{~mm}$ of leaking spot when the internal pressures are $0.25 \mathrm{MPa}$ and $0.45 \mathrm{MPa}$.

1) When the pressure is $0.25 \mathrm{MPa}$, the amplitude of leakage signals is about $3 \mathrm{~V}$. When the pressure is $0.45 \mathrm{MPa}$, the amplitude increases to $5 \mathrm{~V}$. The conclusion reached is that under the same leakage aperture, the amplitude of leakage signals continues to increase versus internal pressure.

2) The frequency of leakage signals mainly concentrates between $0 \sim 300 \mathrm{kHz}$. As the internal pressure increases, more leakage signals are of high frequency.

These two conclusions are in line with the theory introduced in Chapter 2. When the leakage aperture remains unchanged, the higher the pressure is, the higher the noises are and the bigger the amplitude of leakage signals is. And according to theories, when the internal pressure increases, the leakage speed would also raise and leakage signals would be of higher frequency, which results in that more signals are of high frequency than under a low pressure [14-15].
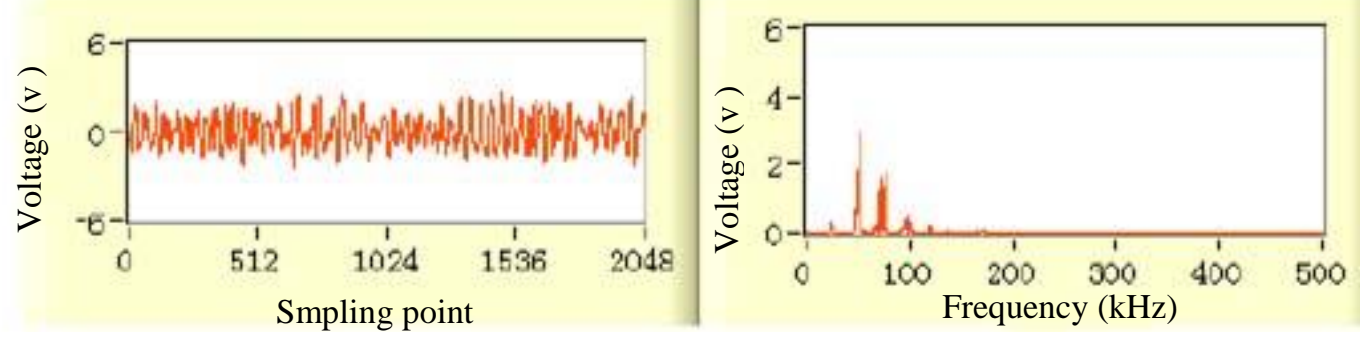

(a) The Internal Pressure is $0.25 \mathrm{Mpa}$
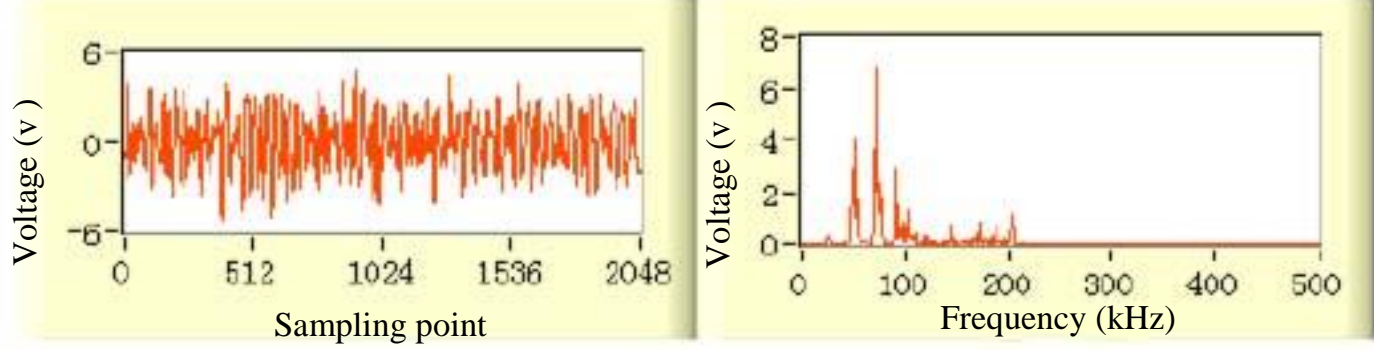

(b) The Internal Pressure is $0.45 \mathrm{Mpa}$

Figure 4. Waveform and Frequency Spectrum of Leakage Signals under Different Pressures 
(3) Variation of waveform and frequency spectrum of leakage signals versus leakage aperture

(a) (e) In Figure 5 are acoustic emission signals at 1, 2, 3, 4 and 5mm of leakage aperture when the internal pressure is $0.25 \mathrm{MPa}$. From the waveform, it can be seen that the leakage signals are constant.

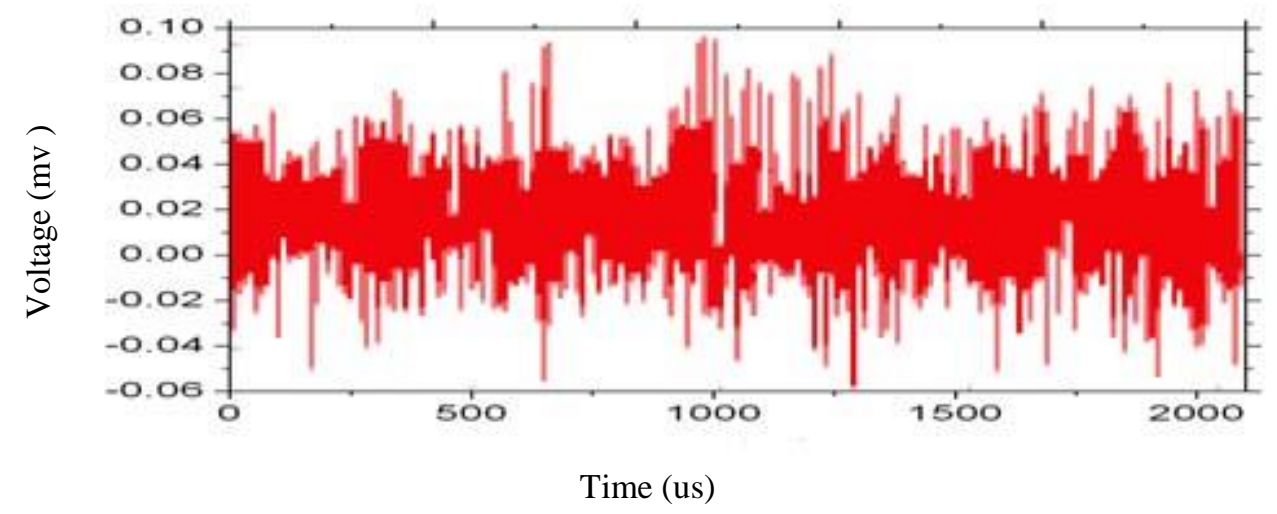

(a) $1 \mathrm{~mm}$ leaking spot

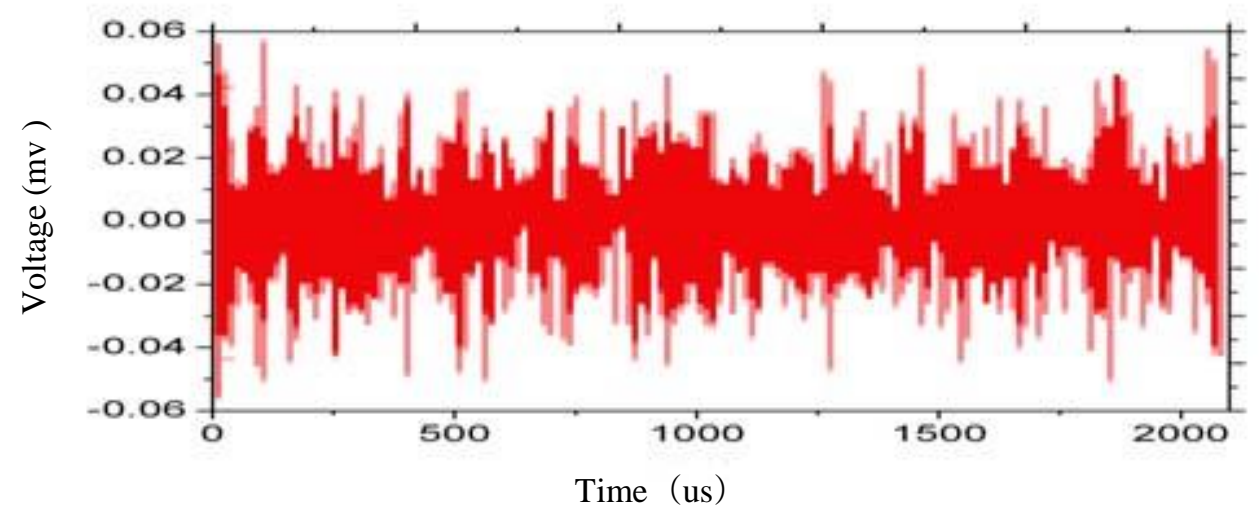

(b) $2 \mathrm{~mm}$ leaking spot

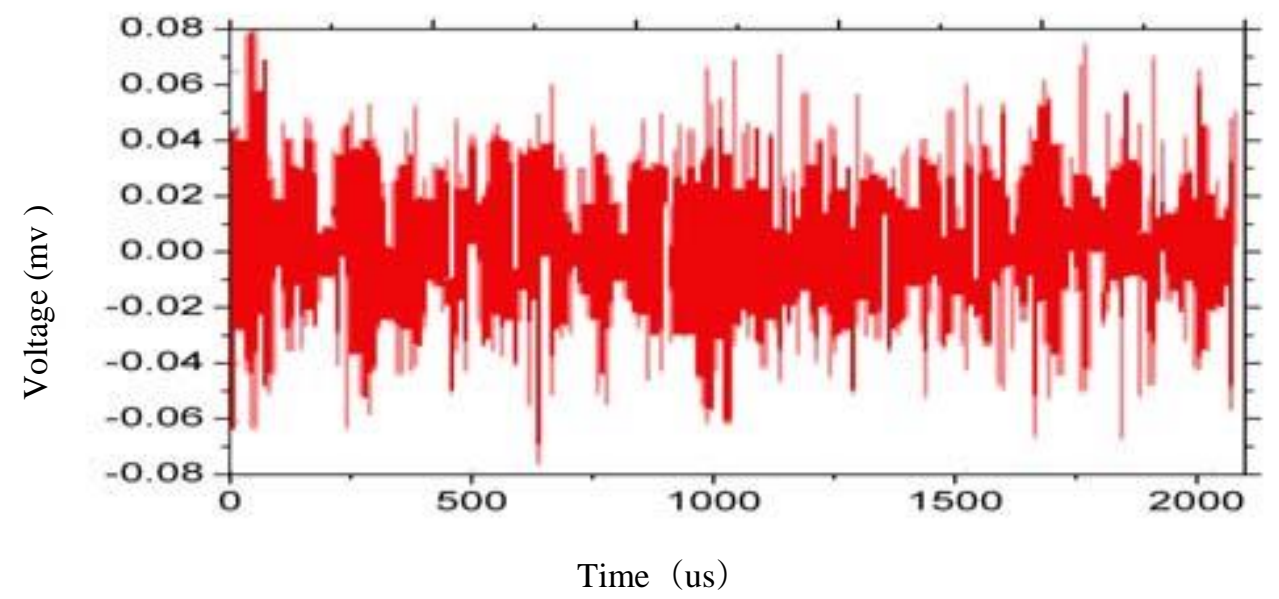

(c) $3 \mathrm{~mm}$ leaking spot 


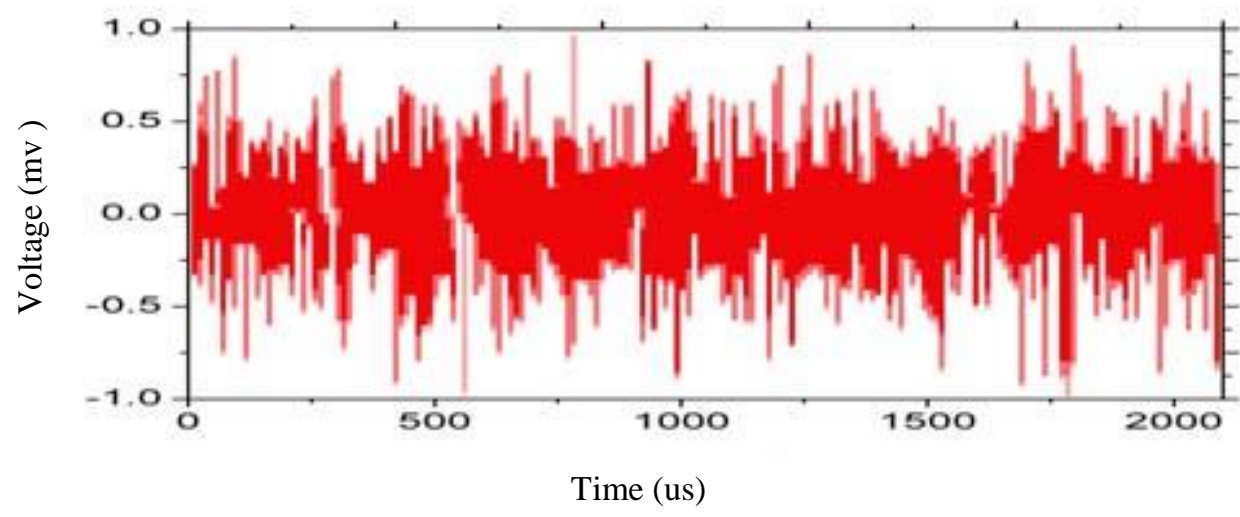

(d) $4 \mathrm{~mm}$ leaking spot

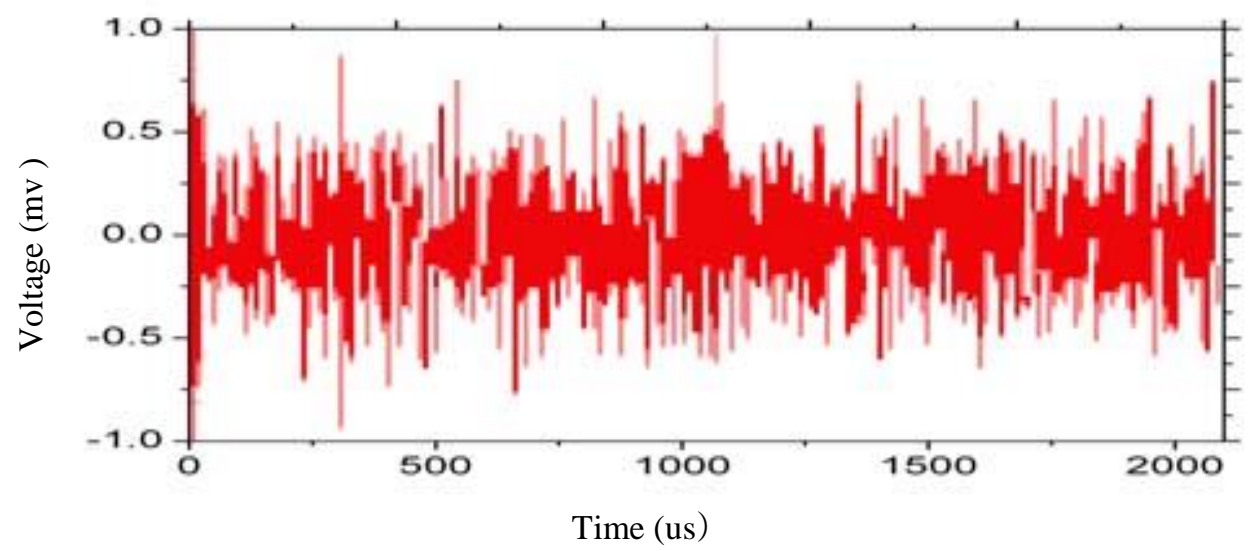

(e) $5 \mathrm{~mm}$ leaking spot

\section{Figure 5. Waveform and Frequency Spectrum of Leakage Signals under Different Leakage Apertures when the Internal Pressure is $0.25 \mathrm{Mpa}$}

Figure 6 Waveform and frequency spectrum of leakage signals under different leakage apertures when the internal pressure is $0.3 \mathrm{Mpa}$, From Figure 6, it is known that:

1) Compare waveforms. The amplitude of signals in two figures is different because of different leakage aperture. A big leakage aperture corresponds to big amplitude, which is proved by the fact that the amplitude in (b) is bigger than in (a).

2) Compare frequency spectrums at different leaking spots. Leakage aperture has great influence on the frequency energy of leakage signals but little influence on the frequency distribution. The frequency of signals mainly concentrates between $0 \sim 300 \mathrm{kHz}$.

These conclusions are in line with theories in Chapter 2. When the internal pressure remains unchanged, sound intensity at different leaking spots is distinct. When the sound is loud, the amplitude of leakage signals is big. As the leakage aperture increases, the maximum leakage speed doesn't change much. Consequently, frequency distribution of leakage signals is hardly affected by leaking spot. 

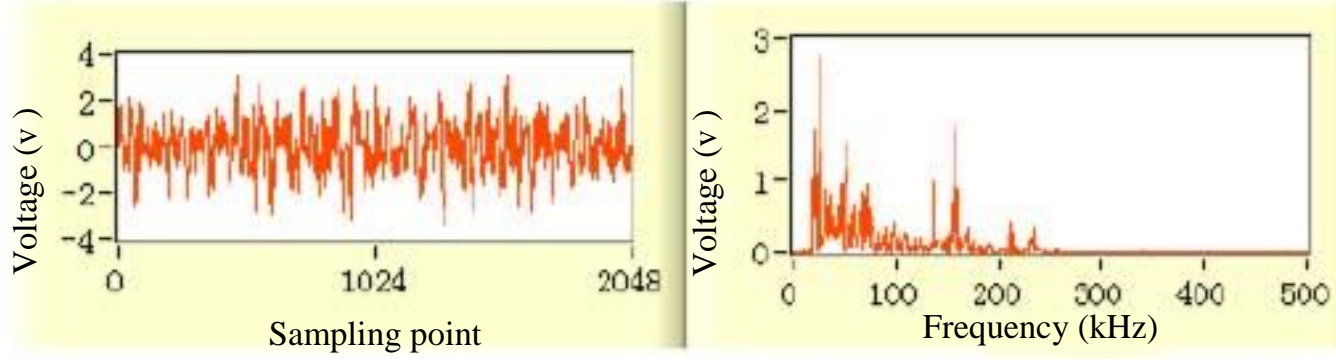

(a) 1 mmleaking spot

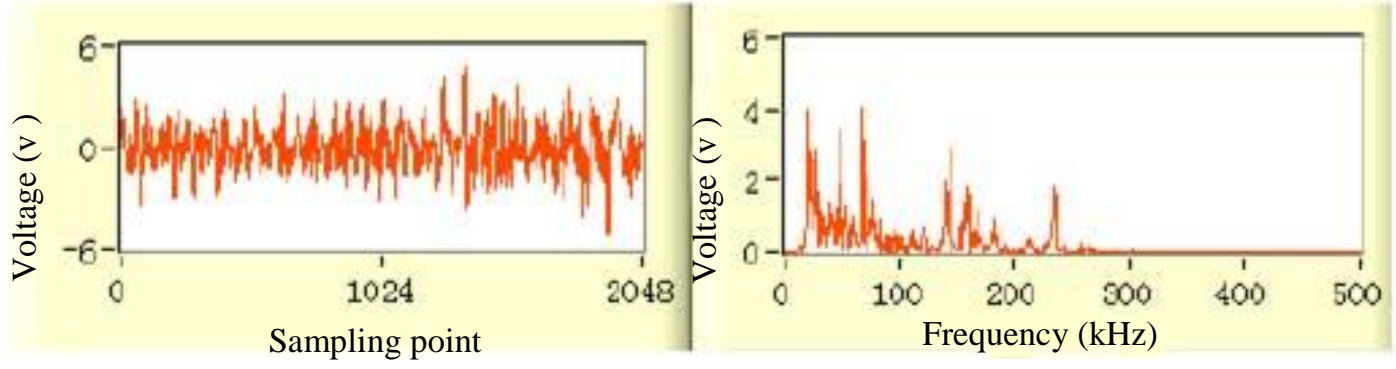

(b) 3mmleaking spot

\section{Figure 6. Waveform and Frequency Spectrum of Leakage Signals under Different Leakage Apertures}

\section{(4) Variation of Leakage Signal Amplitude versus Transmission Distance}

Figure 7 shows the variation of leakage signal amplitude versus transmission distance when the internal pressure is $0.25 \mathrm{MPa}, 0.35 \mathrm{MP}$ and $0.45 \mathrm{MPa}$ at $1 \mathrm{~mm}$ of leaking spot. It can be concluded that as the transmission distance increases, the amplitude of leakage signals gradually reduces.

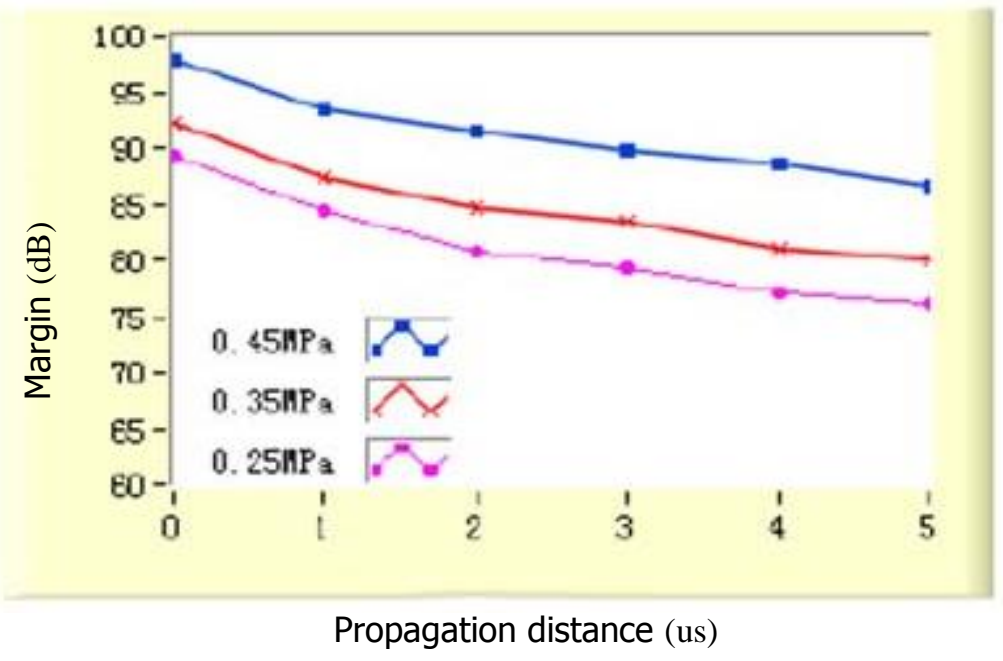

Figure 7. Leakage Signal Amplitude versus Transmission Distance

\section{Conclusion}

Through the experiment, wave, frequency spectrum, amplitude and energy of acoustic emission signals produced from inner pipeline leakage versus pressure, leakage aperture and transmission distance were studied with the removal of the shell through experiment. This paper reached the following conclusions: 
(1) The amplitude and energy of acoustic emission signals of leakage increased versus pressure and leakage aperture, but decreased versus transmission distance;

(2) Energy of acoustic emission signals of leakage mainly concentrated between 0 300 $\mathrm{kHz}$. When the diameter of the leakage aperture was small, some acoustic emission signals were of high frequency. As the leakage aperture increased, signals were mainly of low frequency;

(3) As the internal pressure of the pipeline decreased, the amplitude of acoustic emission signals of leakage continued to decrease. Under different pressures, waves of different frequencies had roughly the same proportion of energy in the total energy;

(4) When the leakage aperture changed, the amplitude and energy of acoustic emission signals of leakage were mainly influenced by the flow rather than by the speed. Consequently, frequency distribution of leakage signals was hardly influenced by the leaking spot;

(5) As the transmission distance increased, the amplitude of leakage signals decreased with signals of high frequency reduced more quickly than signals of low frequency.

\section{Acknowledgements}

Foundation item: Nature Science Foundation of Henan Science and Technology Bureau (141008) (141080); Major science and technology research plan project in Henan province (142102110028).

\section{References}

[1] K. H. Kabir, M. A. Alim and L. Andallah, "Effects of Pressure Work on Mhd Natural Convection Flow along a Vertical Wavy Surface", International Journal of Heat and Technology, vol. 32, no. 1-2, (2014), pp. 95-102.

[2] G. Heo and S. Kyu Lee, "Internal leakage detection for feedwater haters in power plants using neural networks", Expert Systems with Applications, vol. 39, no. 5, (2012) April, pp. 5078-5086.

[3] M. J. Eaton, R. Pullin and K. M. Holford, "Acoustic emission source location in composite materials using Delta T Mapping", Composites Part A: Applied Science and Manufacturing, vol. 43, no. 6, (2012) June, pp. 856-863.

[4] S. Hao, J. Shuguang, W. Lanyun and W. Zhengyan, "King factor of the strata overlying the gob and a three-dimensional numerical simulation of the air leakage flow field", Mining Science and Technology (China), vol. 21, no. 2, (2011) March, pp. 261-266.

[5] S. Ghosh, D. Mukhopadhyay and S. Kumar Saha, "An experimental analysis of subcooled leakage flow through slits from high pressure high temperature pipelines", International Journal of Pressure Vessels and Piping, vol. 88, no. 8-9, (2011) August-September, pp. 281-289.

[6] D. Asher Ghertner and M. Fripp, "Ding away damage: Quantifying environmental leakage through consumption-based, life-cycle analysis", Ecological Economics, vol. 63, no. 2-3, (2007) August 1, pp. 563-577.

[7] W. M. Chambers and N. J. McC. Mortensen, "Toperative leakage and abscess formation after colorectal surgery”, Best Practice \& Research Clinical Gastroenterology, vol. 18, no. 5, (2004) October, pp. 865880.

[8] C.-M. Keum, J.-H. Bae, M.-H. Kim, W. Choi and S.-D. Lee, "Ution-processed low leakage organic field-effect transistors with self-pattern registration based on patterned dielectric barrier", Organic Electronics, vol. 13, no. 5, (2012) May, pp. 778-783.

[9] H. Román-Flores, A. Flores-Franulič and Y. Chalco-Cano, "fuzzy integrals", Applied Mathematics and Computation, vol. 204, no. 1, (2008) October 1, pp. 178-183.

[10] C. Lei and P. Zou, "Application of neural network in heating network leakage fault diagnosis", Journal of Southeast University, vol. 26, no. 2, (2010), pp. 173-176.

[11] M. Rahimi, S. Ehsan Rafiee and N. Pourmahmoud, "Numerical Investigation of the Effect of Divergent Hot Tube on the Energy Separation in a Vortex Tube", International Journal of Heat and Technology, vol. 31, no. 2, (2013), pp. 17-26.

[12] D. H. Zhou, X. He, Z. Wang, G.-P. Liu and Y. D. Ji, "Leakage Fault Diagnosis for an Internet-Based Three-Tank System: An Experimental Study", IEEE Transactions on Control Systems Technology, (2011) June 13. 
[13] E. Tuncay and Y. Obara, "Comparison of stresses obtained from Acoustic emission and Compact Conical-Ended Borehole Overcoring techniques and an evaluation of the Kaiser Effect level", Bulletin of Engineering Geology and the Environment, vol. 71, no. 2, (2012), pp. 367-377.

[14] S. Eshan Rafiee, M. Rahimi and N. Pourmahmoud, "Three-Dimensional Numerical Investigation on a Commercial Vortex Tube Based on an Experimental Model - Part I: Optimization Of The Working Tube Radius", International Journal of Heat and Technology, vol. 31, no. 1, (2013), pp. 49-56

[15] Q. Dai, K. Ng, J. Zhou, E. L. Kreiger and T. M. Ahlborn, "Damage investigation of single-edge notched beam tests with normal strength concrete and ultra-high performance concrete specimens using acoustic emission techniques", Construction and Building Materials, vol. 31, (2012) June, pp. 231-242. 
International Journal of Smart Home

Vol. 9, No. 2 (2015) 